\title{
Development of Exhaled Breath Monitoring System Using Semiconductive Gas Sensors and Statistical Analysis
}

\author{
Toshio Iton ${ }^{1}$, Toshio Miwa ${ }^{1}$, Akihiro Tsuruta1, Takafumi Akamatsu ${ }^{1}$, Noriya Izu', Woosuck Shin', \\ Jangchul Park², Toyoaki Hida², Yuichi Sakumura3,5, Takeshi Eda ${ }^{4}$, Yasuhiro Setoguchi4 \\ ${ }^{1}$ National Institute of Advanced Industrial Science and Technology (AIST), \\ Shimo-shidami, Moriyama-ku, Nagoya 463-8560, Japan, \\ 2 Aichi Cancer Center, 1-1 Kanokoden, Chikusa-ku, Nagoya 464-8681, Japan, \\ ${ }^{3}$ Department of Information Science and Technology, Aichi Prefectural University, \\ Nagakute 480-1198, Japan, \\ ${ }^{4}$ Figaro Engineering Inc., 1-5-11 Sembanishi, Minoh, Osaka 562-8505, Japan, \\ ${ }^{5}$ Present address: Graduate School of Biological Sciences, Nara Institute of Science and Technology, \\ Ikoma, Nara 630-0192, Japan \\ Corresponding author's e-mail address: itoh-toshio@aist.go.jp
}

\begin{abstract}
:
Volatile organic compounds (VOCs) in exhaled breath samples from lung cancer patients (LC) and healthy controls (HC) were analyzed by gas condenser-equipped GC/MS for development of an exhaled breath monitoring prototype system involving gas condensing units, short GC columns, and metal oxide semiconductive gas sensors. The GC/MS analysis identified 56 VOCs in exhaled breath samples from 136 volunteers, 107 LC and $29 \mathrm{HC}$, and selected four target VOCs by simple statistical analyses. The first prototype analyzed exhaled breath samples from 101 volunteers (74 LC and 27 $\mathrm{HC}$ ). The prototype system exhibited a level of performance similar to that of the gas condenserGC/MS system for breath analysis. Subsequently, we developed the second prototype which used advanced statistical analysis, support vector machine algorithm (SVM), for improving high percentage of correct answers. The SVM was also carried out for selected new target VOCs from results of the GC/MS analysis. The short GC columns of the second prototype were selected for detecting the new four target VOCs.
\end{abstract}

Key words: lung cancer, volatile organic compounds (VOCs), exhaled breath, gas chromatography (GC), semiconductive gas sensors.

\section{Introduction}

Monitoring of the exhaled breath is one of the best approach for early diagnosis because of noninvasive screening technique. However, the monitoring of the exhaled breath is limited to use for specific diseases, as a lot of diseaserelated volatile organic compounds (VOCs) are present in the exhaled breath at ppb level.

In this study, we developed a prototype system for screening exhaled breath. The prototype involved gas condensing units for analyzing ppb levels of VOCs, short GC columns for gasselectivity, and noble metal-loaded tin oxide gas sensors as detectors. Biomarker VOCs of lung cancer have been extensively studied. Various compounds have been reported as optimal biomarkers, suggesting differences in VOC profiles among many previous studies. The differences may arise from differences in analytical conditions. We also analyzed exhaled breath samples using a gas condenserequipped GC/MS for development of GCs with gas condenser-type prototype systems.

\section{Experimental}

After approval by the local ethics committee of Aichi Cancer Center and AIST and obtaining written informed consent, 107 patients with lung cancer and 29 healthy controls were enrolled in the gas condenser-equipped GC/MS study. All volunteers blew their alveolar breath into $1 \mathrm{~L}$ of Analytic Barrier bags after they exhaled their respiratory tract air. The gas condenser-GC/MS analysis used a GCMS-QP2010 instrument (Shimadzu, Japan) equipped with a TD-2 gas- 
condensing unit (Shimadzu). The TD-2 aspirated the exhaled breath at $60 \mathrm{~mL} / \mathrm{min}$ for condensation of VOCs in a cold trap at $-20^{\circ} \mathrm{C}$ for $4 \mathrm{~min}$. Then the cold trap was heated and desorbed VOCs for applying to the GC/MS.

The prototype used a gas condensing unit and a double column with detectors for detecting four types of VOCs. Tenax TA was used as the adsorbent agent. The two $\mathrm{Pt}, \mathrm{Pd}$, and $\mathrm{Au}$ loaded $\mathrm{SnO}_{2}$ sensor elements were installed into the prototype as detectors [1,2]. Fig. 1 shows a flow stream of the prototype.

After obtaining approval by the local ethics committee of Aichi Cancer Center and AIST and written informed consent from the participants, 74 patients with lung cancer and 27 healthy controls were enrolled in this study. $200 \mathrm{~mL}$ of exhaled breath was aspirated for condensation of VOCs in Tenax TA. Then the Tenax TA was heated and desorbed VOCs for applying to the two GC columns.

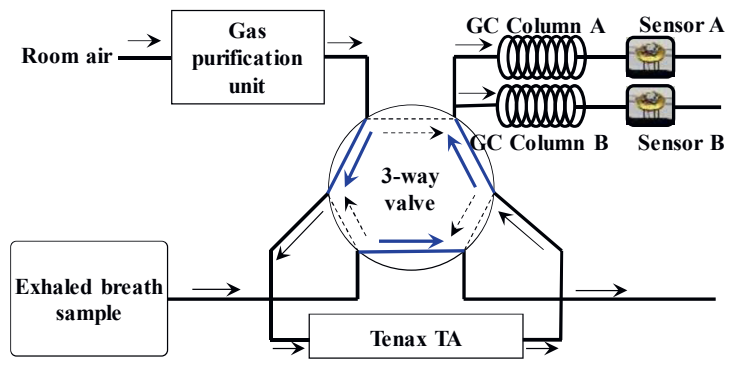

Fig. 1. Flow stream of the prototype.

\section{Results and Discussion}

On the first study, we carried out simple statistical analyses, t-test and correlation coefficient analysis, using results from the gas condenser-GC/MS. We selected four target VOCs, i.e., nonanal, acetoin, acetic acid, and propanoic acid. Fig. 2 shows the GC spectra of the prototype of the exhaled breath from a lung cancer-patient. We evaluated the performance of the prototype by comparison with the results of gas condenser-GC/MS using a percentage of lung cancer-samples that included VOCs at concentrations higher than the maximum concentration from the healthy controls. The prototype and gas condenser-GC/MS analyses detected $13.5 \%$ and $13.1 \%$ of LC samples, indicating that the prototype exhibited similar performance to gas condenser-GC/MS system [3]. However, the sensitivity of $13.5 \%$ is not sufficient for screening.

On the second study, we carried out an advanced statistical analysis, support vector machine (SVM), using results from the gas condenser-GC/MS for high percentage of correct answers. As a result, different four target VOCs were selected. The SVM algorism can detected on $76.5 \%$ accuracy using the four target VOCs. Fig. 3 shows new prototype system using the SVM algorithm.
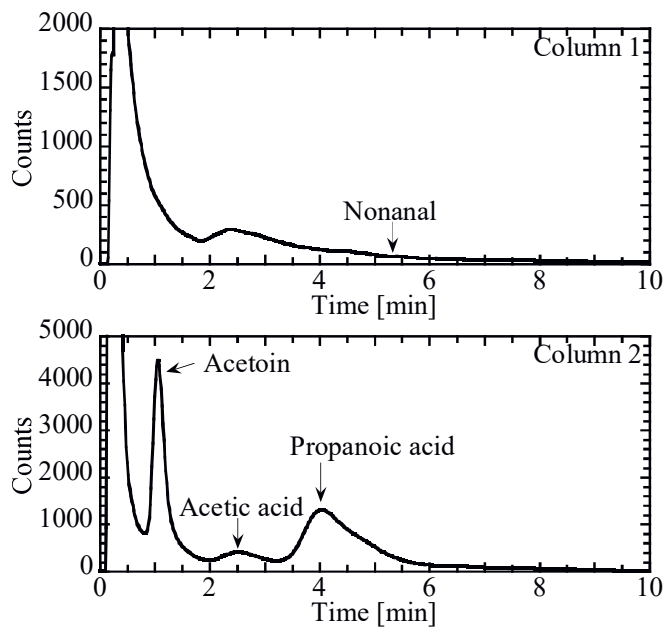

Fig. 2. GC spectra from two columns of the prototype of exhaled breath samples from a lung cancer-patient.

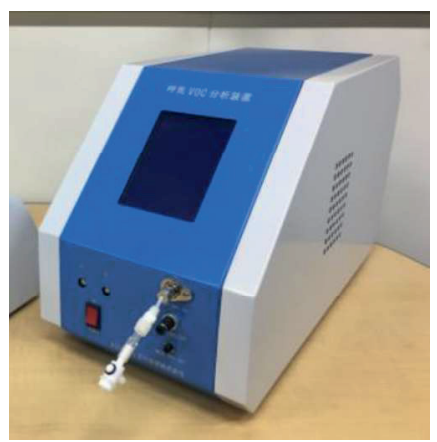

Fig. 3. New prototype for exhaled breath VOCs monitoring with SVM algorithm.

\section{Acknowledgement}

This work was supported in part by the "Development Project for Extremely-Early Diagnostics Technologies for Human Diseases" of Aichi prefecture, Japan. We also express our sincere thanks to Professor Kazuo Sato (Aichi Institute of Technology; group leader of the project) for his kind advice on this study.

\section{References}

[1] T. Itoh, et al., Nonanal gas sensing properties of platinum, palladium, and gold-loaded tin oxide VOCs sensors, Sens. Actuators B 187, 135-141 (2013); doi: 10.1016/j.snb.2012.09.097

[2] T. Itoh, et al., Analysis of Recovery Time of Pt-, $\mathrm{Pd}-$, and $\mathrm{Au}$-Loaded $\mathrm{SnO}_{2}$ Sensor Material with Nonanal as Large-Molecular-Weight Volatile Organic Compounds, Sens. Mater. 28, 11651178 (2016); doi: 10.18494/SAM.2016.1278

[3] T. Itoh, et al., Development of an exhaled breath monitoring system with semiconductive gas sensors, a gas condenser unit, and gas chromatograph columns, Sensors 16, 1891 (2016); doi: 10.3390/s16111891 